\title{
Modeling and Simulation of Arresting Gear System with Multibody Dynamic Approach
}

\author{
Wenhou Shen, ${ }^{1,2}$ Zhihua Zhao, ${ }^{1}$ Gexue Ren, ${ }^{1}$ and Jiapeng Liu ${ }^{1}$ \\ ${ }^{1}$ Tsinghua University, Beijing 100084, China \\ ${ }^{2}$ Naval Aviation Institute, Huludao 125001, China \\ Correspondence should be addressed to Wenhou Shen; shenwenhou@sina.com
}

Received 17 July 2013; Revised 19 September 2013; Accepted 19 September 2013

Academic Editor: Song Cen

Copyright (C) 2013 Wenhou Shen et al. This is an open access article distributed under the Creative Commons Attribution License, which permits unrestricted use, distribution, and reproduction in any medium, provided the original work is properly cited.

\begin{abstract}
The arresting dynamics of the aircraft on the aircraft carrier involves both a transient wave propagation process in rope and a smooth decelerating of aircraft. This brings great challenge on simulating the whole process since the former one needs small time-step to guarantee the stability, while the later needs large time-step to reduce calculation time. To solve this problem, this paper proposes a full-scale multibody dynamics model of arresting gear system making use of variable time-step integration scheme. Especially, a kind of new cable element that is capable of describing the arbitrary large displacement and rotation in three-dimensional space is adopted to mesh the wire cables, and damping force is used to model the effect of hydraulic system. Then, the stress of the wire ropes during the landing process is studied. Results show that propagation, reflection, and superposition of the stress wave between the deck sheaves contribute mainly to the peak value of stress. And the maximum stress in the case of landing deviate from the centerline is a little bit smaller than the case of landing along centerline. The multibody approach and arresting gear system model proposed here also provide an efficient way to design and optimize the whole mechanism.
\end{abstract}

\section{Introduction}

The arresting gear system is an essential unit equipped by aircraft carrier to assist landing of high-speed aircraft within limited distance. Its first prototype was invented in 1911 by Hugh Robinson [1], the rope of which across the deck is grasped and pulled out by the aircraft tailhook, raising sandbags at both ends of the rope via the pulleys and slowing down the aircraft. Afterwards, the hydraulic arresting gears, which replaced the retarding force from the gravity of sandbags to hydraulic damping, appeared [2]. It soon became popular in modern aircraft carriers since the hydraulic force is adjustable to land aircrafts of different weights and velocities with the same mechanism. The most often equipped one is the MK7 type hydraulic arresting gears.

A typical arresting process contains two sequential stages with completely different dynamical characters: the first stage is dominated by the stress wave propagation and reflection within rope, triggered by the impact between pendant and tailhook; the second stage is governed by the quasi-steady hydraulic forces when the stress wave is decayed and followed by a smooth large overall motion of the cable and quick deceleration of the aircraft. The whole process is so complicated that some mechanical problems are hard to answer through theory analysis and even numerical simulation, such as when the maximum stress in rope will happen and where it is, what is the key factor when the aircraft is landing along or deviate from the centerline, how to control the hydraulic forces to make sure that the aircraft is arrested within limited distance accompanying a steady deceleration process. Although many scholars have been devoted on studying different aspects of arresting gear system, there are still many problems concerning the deck pendant which result in several flight accidents nowadays, the most recent of which was the fracture of the ropes at USS George Washington in 2003 [3].

In literatures, Billec [4] tested experimentally the acceleration of aircraft with different deck pendant length in 1967 . He pointed out that the maximum deceleration reduced about 14 percent when the pendant increased from 100 feet to 130 feet. In 2010, Zhuokun et al. [5, 6] analytically calculated the maximum steady tension of the wire rope with quasisteady landing assumption; they conclude that the maximum 
tension in the offline landing case will be about 3.3\% smaller than the centerline landing case. In 2009, Mikhaluk et al. [7] proposed to build the whole arresting gear system with aids of commercial finite element software LS-DYNA. They meshed the cables with beam element, defined the contact between cables and pulleys as Hertz contact model, and developed new hydraulic damping to model the hydraulic forces. The centerline landing cases with an aircraft of a weight of 14 ton and a velocity ranging from $180 \mathrm{~km} / \mathrm{h}$ to $240 \mathrm{~km} / \mathrm{h}$ are simulated, and the achieved hydraulic pressure is similar to that of the experiment data. Later, Xinyu [8] and Lihua et al. $[9,10]$ also adopted the LS-DYNA to construct the whole arresting system, and the cables are modeled with concentrated spring and mass units. They pointed out that the maximum stress appeared at the moment that the stress wave reached the pulley and reflected from the pulley to the tailhook.

Although the LS-DYNA model is competent to simulate the whole arresting process and capture the stress wave dynamics at initial impact stage, the explicit dynamics strategy in LS-DYNA requires both fine mesh for the flexible cables and small time-step to guarantee the stability of integration algorithm. These two facts reduce the efficiency greatly both at the first impact stage and the second steady stage. Besides, the current available studies are focused on the centerline landing case, and no simulation has appeared in literature about the offline landing cases.

Based on the above problems, in this paper a full-scale MK7 model of the whole arresting gear system under the frame of the multibody system dynamics is presented. The governing equations of the whole system are solved through an implicit backward differentiation formula to reduce the simulation time, since it can use larger time-step compared with explicit integration algorithm and the time-step is adjustable according to the dynamical properties of problem. The model includes an aircraft and its tailhook, hydraulic system and dampers, deck pendant, and purchase wire. The cable element is introduced to simulate the arbitrary large displacement and rotation of the wire ropes, and damping force is used to model the effect of hydraulic system. The functionality of hydraulic parts is studied in detail. Furthermore, the stress of the wire ropes in the along and deviate from centerline landing cases are simulated and analyzed. It is found repeatedly that the reflection and superposition of the stress wave at the deck sheave contribute mainly to the peak value of stress. And it draws a conclusion that the maximum stress in the offline landing case is a little bit smaller than the along centerline landing case.

The following context is organized in this way. In Section 2, we build the arresting gear system model through multibody system dynamics approach, verify some elements at Section 3, and simulate the full scale MK7 type arresting gear system at Section 4. Conclusions are given in Section 5.

\section{Modeling MK7 Type Arresting Gear System}

The basic structure and working principle of MK7 type arresting gear system are shown in Figure 1. During the deck landing, the aircraft tailhook grasps the deck pendent (1) which is a cross the deck. The pendent is connected through muffles
(2) with two purchase cables (3) on its both ends. Each purchase cable is reeved through the fixed (5) and movable (8) carriage of one group, and the endpoint is connected by the anchor damper (9). The movable carriage is forced to run toward the fixed carriage when the rope is pulling out by aircraft. And the hydraulic oil in the main cylinder (6) flows out since the plunger (7) of the hydraulic cylinder is fixed with the movable carriage. The flow is separated into two branches. The main one goes through the constant runout control valve (10) whose open area is passively adjusted during the landing processes to produce a steady retraction force for slowing down the aircraft. The minor one goes to the anchor dampers (9) to provide damping forces for absorbing shocks and vibrations of purchase cable. Besides, when the tailhook of the aircraft grasps the deck pendant at initial stage, there appears a severe vibration of the rope. Two damper sheave installations (4) are introduced to reduce the amplitude of the vibration in deck pendent.

Obviously, the arresting gear system is a complicated mechanical and hydraulic coupled system which contains rigid bodies, flex ropes, and hydraulic units. All of the them will be modeled here through multibody system dynamic approach as illustrated in Figure 2. And the governing equation of each part will be discussed in the left context of this section.

2.1. Governing Equations of Rigid Bodies. The landing aircraft, deck sheaves, fixed and movable carriage, plunger of the hydraulic cylinder, and muffles are modeled as rigid bodies. Generally speaking, a rigid body undergoing arbitrary large three-dimensional motion owns six, three translational and three rotational, degrees of freedom. In the multibody dynamic approach, the mass center $\mathbf{r}_{\text {rigid }}=[x, y, z]^{T}$ in Cartesian space is usually adopted to describe the translational motion, where the superscript $T$ represents transpose transformation of vector or matrix hereafter. However, there are various ways to parameterize three-dimensional finite rotation, such as Euler angles or rotation vector with three variables and Euler parameters with four variables. Here, we use Euler parameters $\lambda_{\text {rigid }}=\left[\lambda^{0}, \lambda^{1}, \lambda^{2}, \lambda^{3}\right]^{T}$ since it is singularity free in contrast to each three-parameters parameterization of finite rotation that involves singularity problem as proved by [11] and reviewed by [12]. The definition of Euler parameters is based on Euler's rotation theorem, saying that any rotation movement of a rigid body, or composition of them, can be achieved by rotation by an angle $\theta$ with respect to an axis $\mathbf{n}$. And the Euler parameters are defined as

$$
\begin{aligned}
\lambda^{0}=\cos \left(\frac{\theta}{2}\right), & \lambda^{1}=n_{x} \sin \left(\frac{\theta}{2}\right), \\
\lambda^{2}=n_{y} \sin \left(\frac{\theta}{2}\right), & \lambda^{2}=n_{z} \sin \left(\frac{\theta}{2}\right),
\end{aligned}
$$

where $n_{x}, n_{y}$, and $n_{z}$ are the three components of the rotation axis $\mathbf{n}$ and $\theta$ is the rotation angle. The four Euler parameters are not independent, satisfying the following normalization constraint equation

$$
\lambda_{\text {rigid }}^{T} \lambda_{\text {rigid }}-1=0
$$




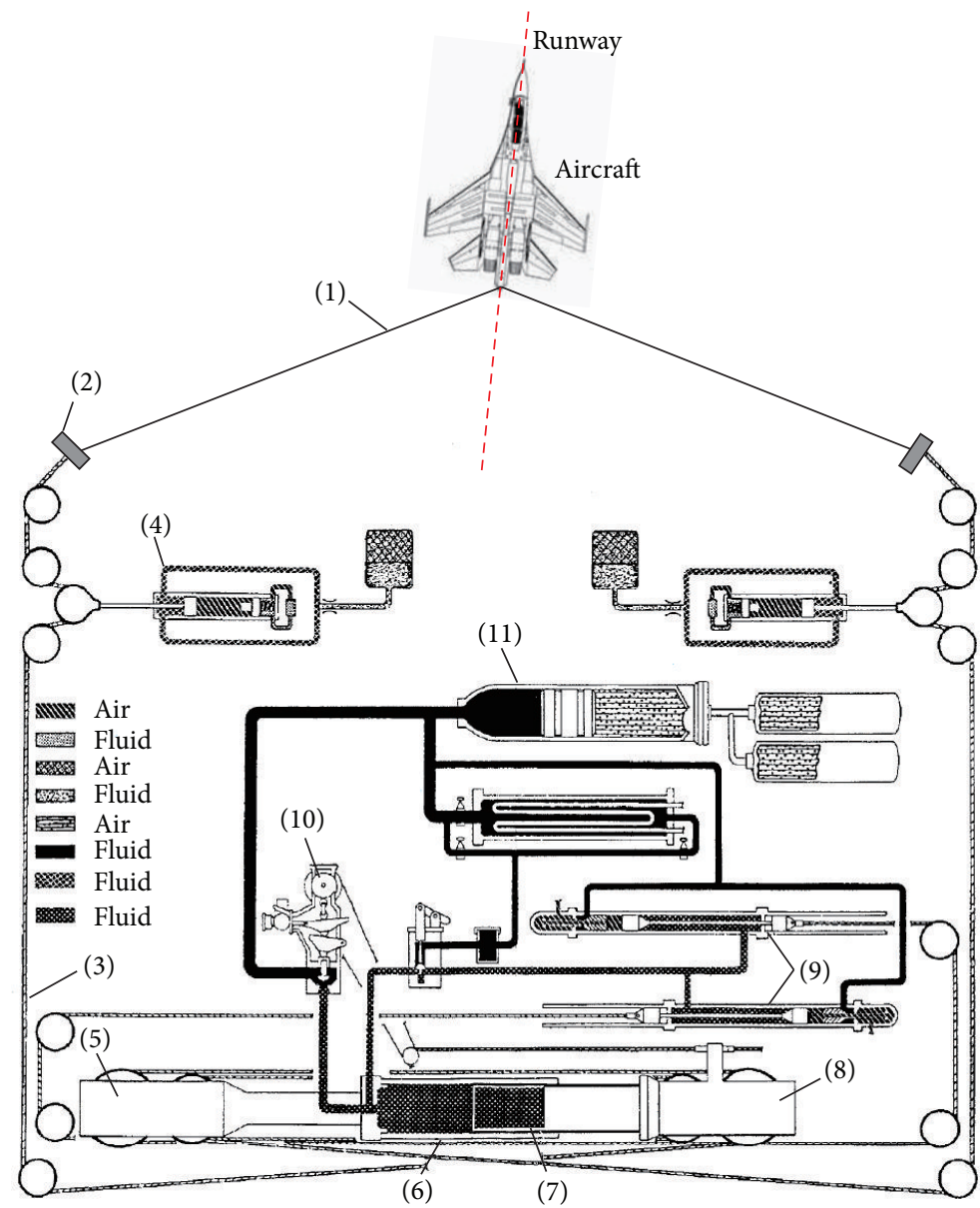
(1) Deck pendant
(7) Plunger of hydraulic cylinder
(2) Connecting muffle
(8) Movable carriage
(3) Purchase cable
(9) Cable anchor damper
(4) Deck sheave installation
(10) Constant runout control valve
(5) Fixed carriage
(11) Accumulator
(6) Hydraulic cylinder

FIGURE 1: Structure and working principle of MK7 type arresting gear system.

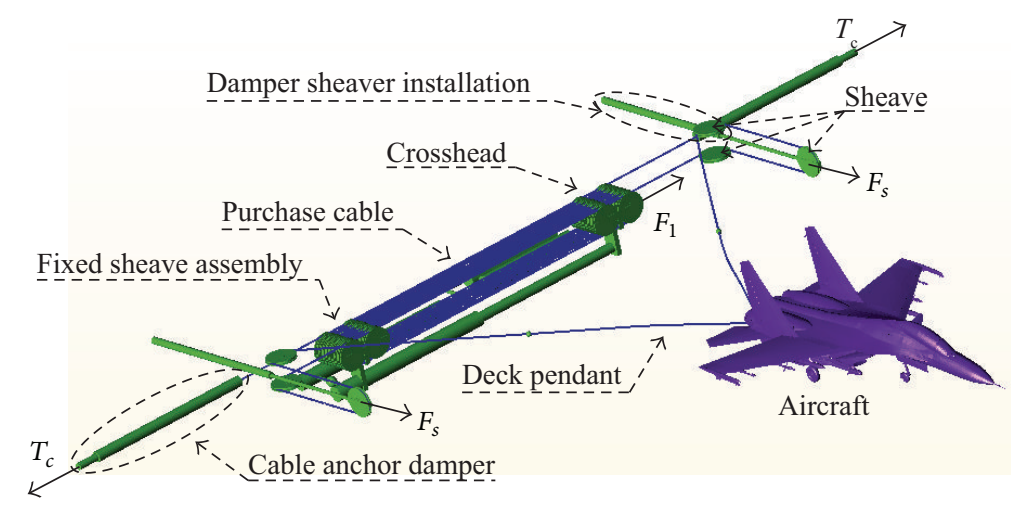

FIGURE 2: Multibody model of the arresting gear system. 
The mass center $\mathbf{r}_{\text {rigid }}$ and Euler parameter $\lambda_{\text {rigid }}$ form the generalized coordinates of a rigid body as

$$
\mathbf{q}_{\text {rigid }}=\left[\mathbf{r}_{\text {rigid }}^{T}, \lambda_{\text {rigid }}^{T}\right]^{T} .
$$

Assume that the whole arresting gear system contains $n_{b}$ rigid bodies and they are interconnected through $n_{c}$ constraints which can be expressed in the following unified form

$$
\Phi_{k}\left(\mathbf{q}_{1}, \mathbf{q}_{2}, \ldots, \mathbf{q}_{n_{b}}, t\right)=0 \quad k=1, \ldots, n_{c} .
$$

Then the governing equations of the whole system will be

$$
\begin{gathered}
\mathbf{M}_{i} \ddot{\mathbf{q}}_{i}+\mathbf{Q}_{i}+\mathbf{F}_{i}+\sum_{k=1}^{n_{c}} \Phi_{k, \mathbf{q}_{i}}^{T} \mu_{k}=0 \quad i=1,2, \ldots, n_{b} \\
\Phi_{k}=0 \quad k=1,2, \ldots, n_{c}
\end{gathered}
$$

derived from the first kind Lagrange equations, where $\mu_{k}$ is the Lagrange multiplier corresponding to constraint $\Phi_{k}, \Phi_{k, \mathbf{q}_{i}}$ is the partial derivative of $\Phi_{k}$ with respect to $\mathbf{q}_{i}$, and

$$
\begin{gathered}
\mathbf{M}_{i}=\left[\begin{array}{cc}
m_{i} \mathbf{I}_{3 \times 3} & 0 \\
0 & \mathbf{B}_{i}
\end{array}\right], \quad \mathbf{B}_{i}=4 \mathbf{G}_{i}^{T} \mathbf{J}_{i} \mathbf{G}_{i}, \\
\mathbf{G}_{i}=\left[\begin{array}{cccc}
-\lambda_{i}^{1} & \lambda_{i}^{0} & \lambda_{i}^{3} & -\lambda_{i}^{2} \\
-\lambda_{i}^{2} & -\lambda_{i}^{3} & \lambda_{i}^{0} & \lambda_{i}^{1} \\
-\lambda_{i}^{3} & \lambda_{i}^{2} & -\lambda_{i}^{1} & \lambda_{i}^{0}
\end{array}\right], \\
\mathbf{Q}_{i}=\left(\mathbf{V}_{i}-\frac{1}{2} \mathbf{V}_{i}^{T}\right) \dot{\mathbf{q}}_{i}, \quad \mathbf{V}_{i}=\frac{\partial\left(\mathbf{M}_{i} \dot{\mathbf{q}}_{i}\right)}{\partial \mathbf{q}_{i}},
\end{gathered}
$$

where $m_{i}$ and $\mathbf{J}_{i}$ are the mass and inertia tensor of the $i$ th rigid body and $\mathbf{I}_{3 \times 3}$ is a $3 \times 3$ identity matrix; the generalized force $\mathbf{F}_{i}$ contains all of the contribution from external forces such as gravity and contact forces.

2.2. Governing Equations of Flexible Cables. During the arresting process, the deck pendant and purchase cable are stretched and bended accompanying large displacement movement. To modele a cable with circular cross-section for multibody dynamics, Shabana $[13,14]$ and several pioneers have proposed absolute nodal coordinate formulation, in which the global position and slope of cable are adopted as generalized coordinates to interpolate the displacement field and calculate the normal strain and bending curvatures. Although this formulation is capable of modeling cables in arresting system, we prefer to introduce nodal position only as generalized coordinate to reduce the calculation scale since the bending energy is negligible. As shown in Figure 3, consider a cable element with a circular cross section area $A$ and length $L$. It contains three uniformly located nodes $N_{1}$, $N_{2}$, and $N_{3}$ with positions $\mathbf{r}_{1}, \mathbf{r}_{2}$, and $\mathbf{r}_{3}$ under the global coordinate system OXYZ. Then the arc length $l$ equals to 0 at $N_{1}, 0.5 L$ at $N_{2}$, and $L$ at $N_{3}$. A normalized elemental parameter $s=l / L$ is introduced for mathematical simplicity. The cable is subjected to a distributed or concentrated external load $\mathbf{f}(s, t)$ which is a function of both space $s$ and time $t$. The governing equation of this element will be derived hereafter.

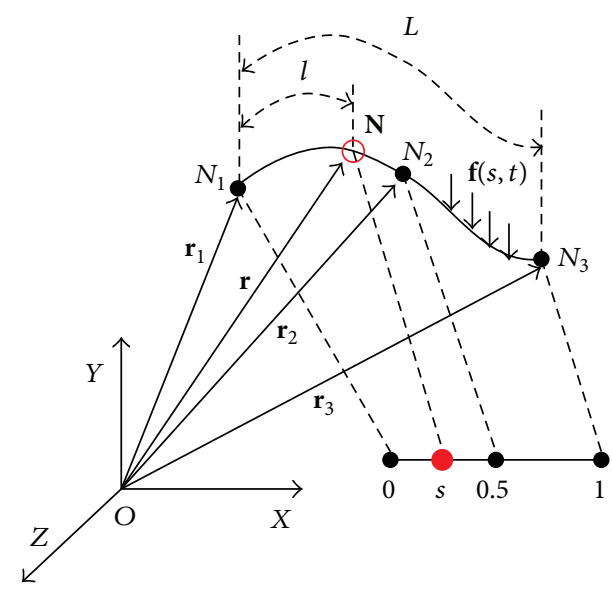

Figure 3: Cable element.

The generalized elemental coordinate $\mathbf{q}_{e}$ is the direct union of three nodal positions, or

$$
\mathbf{q}_{e}=\left[\mathbf{r}_{1}^{T}, \mathbf{r}_{2}^{T}, \mathbf{r}_{3}^{T}\right]^{T} \text {. }
$$

Lagrange functions are introduced as the shape functions to interpolate the elemental position filed. The position vector $\mathbf{r}$ at $s$ is

$$
\mathbf{r}(s, t)=\mathbf{N}(s) \mathbf{q}_{e}
$$

with $\mathbf{N}(s)=\left[N_{1} \mathbf{I}_{3 \times 3}, N_{2} \mathbf{I}_{3 \times 3}, N_{3} \mathbf{I}_{3 \times 3}\right]$, where

$$
\begin{gathered}
N_{1}(s)=2\left(s-\frac{1}{2}\right)(s-1), \\
N_{2}(s)=-4 s(s-1), \\
N_{3}(s)=2 s\left(s-\frac{1}{2}\right) .
\end{gathered}
$$

Then the normal strain of the cable element is

$$
\varepsilon=\frac{1}{2 L^{2}}\left(\mathbf{r}^{\prime T} \mathbf{r}^{\prime}-1\right)
$$

according to Green's strain definition in the theory of elasticity [15], where the prime represents the partial derivative with respect to parameter $s$ or

$$
\mathbf{r}^{\prime}=\frac{\partial \mathbf{r}}{\partial s}=\mathbf{N}^{\prime}(s) \mathbf{q}_{e}
$$

A simple elastoplastic constitutive equation is adopted here to introduce damping force for elastic deformation only, which means that the stress $\sigma$ is

$$
\sigma=E(\varepsilon+\beta \dot{\varepsilon})
$$

where $E$ is the Yong's modulus, $\beta$ is the damping ratio of material, and the overdot represents the partial derivative with respect to time $t$.

Then the virtual work done by inertial force, internal elastic and damping force, and external forces should be zero, which means that

$$
L \int_{0}^{1}\left(-\rho A \ddot{\mathbf{r}}^{T} \delta \mathbf{r}-A \sigma \delta \varepsilon+\mathbf{f}(s, t)^{T} \delta \mathbf{r}\right) \mathrm{d} s=0,
$$




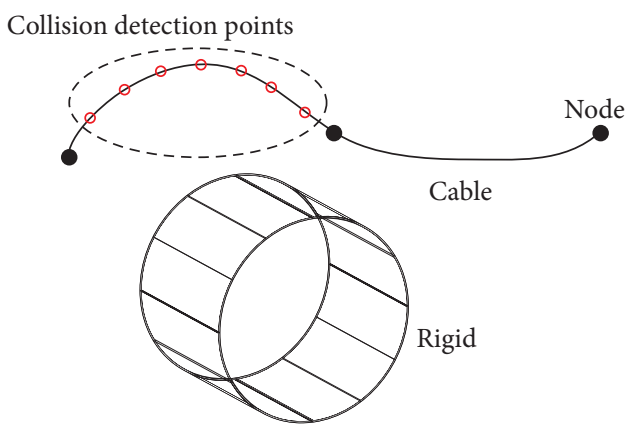

FIgURE 4: Contact detecting between cable and rigid body.

where symbol $\delta$ represents the variation operation. After simplifying, we got the governing equation of the element

$$
\mathbf{M}_{e} \ddot{\mathbf{q}}_{e}+\mathbf{K}_{e}\left(\mathbf{q}_{e}, \dot{\mathbf{q}}_{e}\right) \mathbf{q}_{e}=\mathbf{F}_{e}
$$

with

$$
\begin{gathered}
\mathbf{M}_{e}=\rho A L \int_{0}^{1} \mathbf{N}^{T} \mathbf{N} \mathrm{d} s, \quad \mathbf{K}_{e}=E A L \int_{0}^{1}(\varepsilon+\beta \dot{\varepsilon}) \mathbf{N}^{\prime T} \mathbf{N}^{\prime} \mathrm{d} s, \\
\mathbf{F}_{e}=L \int_{0}^{1} \mathbf{N}^{T} \mathbf{f}(s, t) \mathrm{d} s .
\end{gathered}
$$

It is interesting to note that the elemental mass matrix $\mathbf{M}_{e}$ is a constant matrix and the elemental stiffness matrix $\mathbf{K}_{e}$ vanish once the strain and the time derivative of strain are zeros, which means that cable has stiffness only when it is prestressed.

2.3. Contact between Cable and Rigid Body. According to the assumption of the cable elements, cable is simplified to an axial line in the process of collision between cable and rigid body. When some detecting points are set on the surface of the rigid body and the axial line of the cable, we transform the detection between cable and rigid body to the detection between rigid body and points, as is illustrated in Figure 4 .

According to the Hertz contact theory, the contact force $\mathbf{f}_{c}$ can be expressed as

$$
\mathbf{f}_{c}=f_{n} \mathbf{n}_{f}+f_{t} \mathbf{t}_{f},
$$

where $\mathbf{n}_{f}$ is the unit normal vector of the contact surface; $\mathbf{t}_{f}$ is the corresponding unit tangential vector; $f_{n}$ is the normal collision force; $f_{t}$ is the tangential friction force.

Collision force is exerted on the nodes of the cable element. Therefore, the generalized collision force can be expressed below according to the virtual work principle:

$$
\mathbf{Q}_{p}=\mathbf{N}^{T}\left(s_{n}\right) \mathbf{f}_{n},
$$

where $\mathbf{N}$ is the shape function of the cable element; $s_{n}$ is the element parameter of the detecting point; $\mathbf{f}_{n}$ is the force exerted on the detecting point.
2.4. Hydraulic Forces. To reduce the stress peak value of the deck pendant and vibration of the purchase cable and eliminate the phenomenon of slacking of the rope, damper sheave is installed between two deck sheaves and anchor damper is set on the end of the rope, as is shown in Figure 5.

2.4.1. Damper Sheave Installation. The throttle of the damper sheave installation has an invariable cross section area aperture to let the oil flow goes through, producing the oil damping force. The relationship between damping force and the velocity of the piston motion is as follows [16]:

$$
F_{s}=k_{s} v_{s}^{2}+A_{s} P_{s},
$$

where $k_{s}$ is the equivalent damping coefficient of the oil, $v_{s}$ is the velocity of the piston, $A_{s}$ is the cross section area of the piston, and $P_{s}$ is the pressure of the gas in the air flask.

Omitting the variation of the volume of the oil, the displacement of the piston in the sheave damper is defined as $u_{s}$. As the gas experiences the isentropic process, $P_{s}$ satisfies the relationship as follows

$$
P_{s}=P_{s 0}\left(\frac{V_{s 0}}{V_{s 0}+A_{s} u_{s}}\right)^{\gamma},
$$

where $P_{s 0}$ is the initial pressure of the air flask, $V_{s 0}$ is the initial volume of the air flask, and $\gamma$ is the adiabatic coefficient of the gas.

2.4.2. Cable Anchor Damper. During the arrestment of the aircraft, the cylinder of the cable anchor damper is connected with the main cylinder, so the pressure is equal for the two parts. However, the oil pressure of cylinder is equal to that of air of accumulator in the process of ram going back to its original position.

The cross section area of piston of the cable anchor damper is $A_{c}$, the mass of the piston is $m_{c}$, its displacement increment is $x_{c}$, and the force of the cable anchor damper is $T_{c}$; it can be obtained that

$$
T_{c}=m_{c} \ddot{x}_{c}+P_{c} A_{c}
$$

where $P_{c}$ is the pressure of the oil in the cylinder of the cable anchor damper.

2.4.3. Main Engine Cylinder. When the aircraft is landing, the kinetic energy of the aircraft is transferred to the arresting system by the purchase cable, forcing the ram in the main engine holding the pressurized hydraulic fluid, and is changed to the heat of the hydraulic oil. Therefore, we can apply a damping force on the ram to simulate this procedure.

The main engine cylinder and the ram are illustrated in Figure 6, the hydraulic pressure of the oil in the main engine cylinder is $P_{1}$, the effective cross section area of the ram is $A_{1}$, the mass of the ram and crosshead is $m_{1}$, the sliding velocity of the ram is $v_{1}, F_{1}$ is the force applied on the ram by the arresting rope, and $\mu$ is the damping ratio of the hydraulic oil. It can be gained that

$$
F_{1}=m_{1} \frac{d v_{1}}{d t}+\mu v_{1}+A_{1} P_{1}
$$




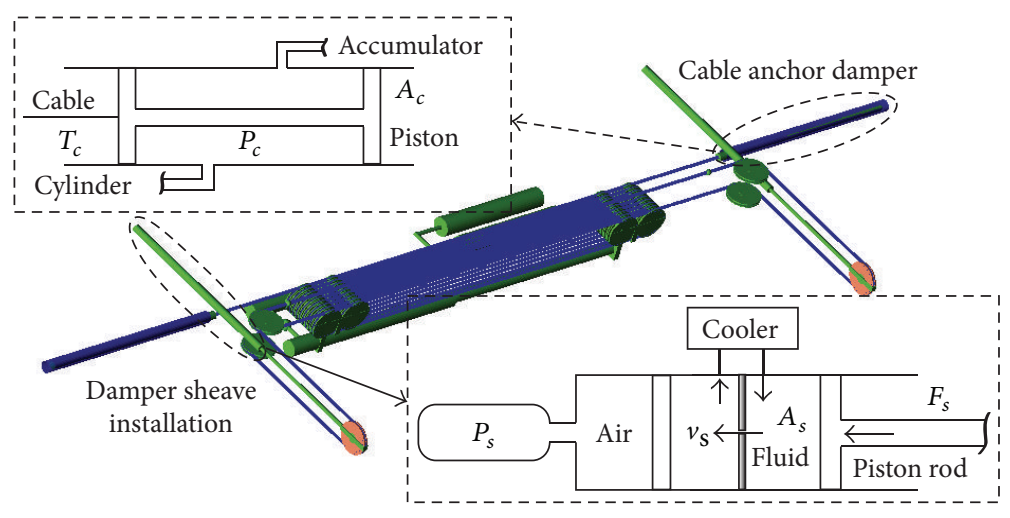

FIGURE 5: Damping mechanism in the arresting gear system.

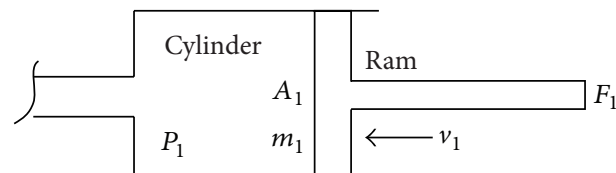

FIgURE 6: Main engine cylinder.

The velocity of the oil in the main engine cylinder is equal to the sliding velocity of the ram; $P_{1}$, which is the hydraulic pressure in the main engine cylinder, is related to the design of the constant runout control valve. If the proposal of the small aperture is adopted [16], the pressure is as follows:

$$
P_{1}=\frac{\rho A_{1}^{2}}{2 c_{d}^{2} A_{2}^{2}} v_{1}^{2}+P_{2},
$$

where $c_{d}$ is the coefficient of the flow which is related to the area of the aperture and the mass of liquid, and so forth, $A_{2}$ is the cross section area of the hole of the throttle, and $P_{2}$ is the pressure of the energy accumulator.

2.4.4. Constant Runout Valve. Constant runout valve is located between the main engine cylinder and the accumulator, controlling the arresting force to arrest the aircraft within a prescribed distance in that it can adjust the increment of the oil in the main engine cylinder by changing the area of the hole of the throttle. Constant runout valve is composed of cam, valve stem, plunger, and the control valve drive system, as is illustrated in the Figure 7.

The cross section area of the hole of the throttle is $A_{2}$, whose relationship with the position of the valve stem $x$ is as follows:

$$
A_{2}=\pi d x \sin \phi\left(1-\frac{x}{2 d} \sin 2 \phi\right),
$$

where $\phi$ is half of the top angle of valve stem; $d$ is the diameter of the valve which connects the main oil cylinder and the throttle, and the relationship between diameter and the $\alpha$ which is the rotation angle of the cam is as follows:

$$
x=x_{0}\left(1-k_{1} \alpha-k_{2} \alpha^{2}\right),
$$

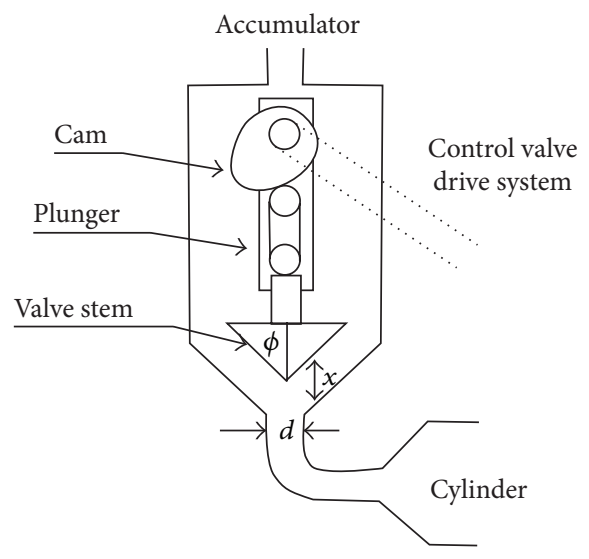

FIGURE 7: Constant runout control valve.

where $k_{1}$ and $k_{2}$ are the functions describing the shape of the surface of the cam; $x_{0}$ is initial area of the throttle whose relationship with diameter of the valve is as follows:

$$
x_{0}=\frac{d}{k_{m}},
$$

where $k_{m}$ is the weight adjusting coefficient. We can change the initial area of the throttle by changing $k_{m}$, so the arresting gear system can capture the aircrafts of different weights.

The control valve drive system is installed between crosshead and the fixed sheaves, so the relationship between the position of the crosshead $u$ and the rotation angle of the cam of the throttle $\alpha$ is as follows:

$$
\alpha=\frac{u}{k_{u}},
$$

where $k_{u}$ is the transfer coefficient between crosshead position and the rotation angle of cam. When the association of the area of the throttle and the position of the crosshead is established, the arresting distance of the aircraft can be controlled.

2.4.5. Integration Method of the Whole System. Based on the above description, the governing equations of the arresting 
gear system form a set of differentiation and algebraic equations (DAEs). They can be generally and uniformly expressed as

$$
\mathbf{E}(t, \mathbf{q}, \dot{\mathbf{q}}, \ddot{\mathbf{q}})=0,
$$

where $\mathbf{q}$ is a ne-dimensional vector of variables, to be solved in the whole system, including rigid bodies' position, Euler parameters, cable node position, and Lagrangian multipliers of constraints, $\mathbf{E}$ is the implicit equations with same dimension ne. One popular numerical algorithm for solving DAEs with contact problems is the implicit first-order backward differentiation formula (BDF). Although the details of firstorder BDF could be found in Shampine and Reichelt's paper [17] or Hairer and Wanner's monograph [18], it is preferred to outline the basic idea here for the integrity of this paper.

Assume that we are going to solve $\mathbf{q}_{n+1}$ at time $t_{n+1}$, which satisfies

$$
\mathbf{E}\left(t_{n+1}, \mathbf{q}_{n+1}, \dot{\mathbf{q}}_{n+1}, \ddot{\mathbf{q}}_{n+1}\right)=\mathbf{0} .
$$

Given the values of $\mathbf{q}$ at time $t_{n}$ and $t_{n-1}$ as $\mathbf{q}_{n}$ and $\mathbf{q}_{n-1}$, the first and second time derivative of $\mathbf{q}_{n+1}$ could be derived through numerical discrete as

$$
\begin{gathered}
\dot{\mathbf{q}}_{n+1}=\frac{1}{h} \mathbf{q}_{n+1}+\boldsymbol{\alpha}, \quad \boldsymbol{\alpha}=-\frac{1}{h} \mathbf{q}_{n}, \\
\ddot{\mathbf{q}}_{n+1}=\frac{1}{h^{2}} \mathbf{q}_{n+1}+\boldsymbol{\beta}, \\
\boldsymbol{\beta}=\frac{1}{h}\left(\frac{1}{t_{n}-t_{n-1}}-\frac{1}{h}\right) \mathbf{q}_{n}+\frac{1}{h} \frac{1}{t_{n}-t_{n-1}} \mathbf{q}_{n-1},
\end{gathered}
$$

where $h=1 /\left(t_{n+1}-t_{n}\right)$ is the current time-step. After substituting (29) into (28), the DAEs are simplified to nonlinear algebraic equations of variables $\mathbf{q}_{n+1}$ only

$$
\mathbf{E}\left(t_{n+1}, \mathbf{q}_{n+1}, \frac{1}{h} \mathbf{q}_{n+1}+\boldsymbol{\alpha}, \frac{1}{h^{2}} \mathbf{q}_{n+1}+\boldsymbol{\beta}\right)=\mathbf{0}
$$

since both $\boldsymbol{\alpha}$ and $\boldsymbol{\beta}$ are known. This nonlinear equation (30) can be solved in terms of the classical Newton-Raphson iteration method, and one possible initial guess $\mathbf{q}_{n+1}^{0}$ of $\mathbf{q}_{n+1}$ for iteration could be get by assuming that $\mathbf{q}$ is linear variable and extending $\mathbf{q}_{n}$ and $\mathbf{q}_{n-1}$ to time $t_{n+1}$

$$
\mathbf{q}_{n+1}^{0}=\mathbf{q}_{n}+\frac{\mathbf{q}_{n}-\mathbf{q}_{n-1}}{t_{n}-t_{n-1}} h .
$$

There are three cases in which the current time-step $h$ will be adjusted.

(1) If the Newton-Raphson iteration fails to get a converged solution, then the $h$ will be reduced and the nonlinear equation (30) will be resolved.

(2) If the Newton-Raphson iteration successfully gives a converged solution $\mathbf{q}_{n+1}^{*}$ and the integration tolerance $\tau_{i}$ is larger than the settled tolerance $\tau$ or $\tau_{i}>\tau$, then the current time-step $h$ will be reduced, where

$$
\tau_{i}=\max \left(\left|\frac{\mathbf{q}_{n+1}^{*}-\mathbf{q}_{n+1}^{0}}{\mathbf{q}_{n}}\right|\right) \frac{1}{h^{2}} .
$$

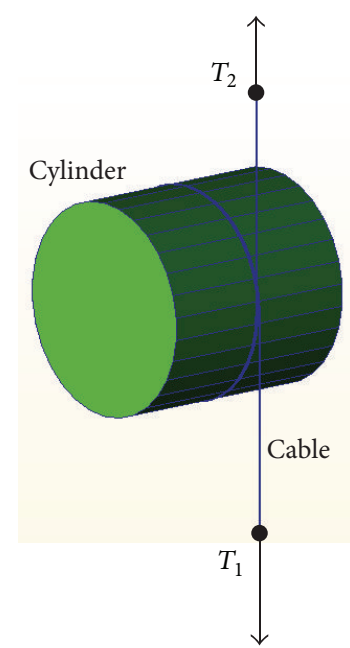

Figure 8: Model of a cable wrapped around a fixed cylinder.

(3) If the Newton-Raphson iteration success with solution $\mathbf{q}_{n+1}^{*}$ the integration tolerance $\tau_{i}$ is one order smaller than the settled tolerance $\tau$ or $\tau_{i}<\tau / 10$, then the current step size will be increased.

In this way, the time-step of BDF is automatically adjusted to fit the dynamic properties of the system. The integration method will chose small time-step for the initial impact stage at arresting process and large time-step for the stable deceleration stage. This saves calculation time a lot compared with fixed time-step integration method.

\section{Verification Example}

In order to verify the established cable element and the collision between the cable and the rigid body, a simple multibody example is presented. As shown in Figure 8, considering a cable wrapped around a fixed cylinder with two full circles, it is subjected to vertical forces $T_{1}$ and $T_{2}$ at two ends, respectively. The friction coefficient between cable and cylinder is $v=0.1$. Then the analytical solution shows that the cable will be balanced when $T_{2}=T_{1} / e^{4 \pi \nu}$. The velocity response of the node at the upper end under the external forces $T_{1}=40 \mathrm{~N}$ and $T_{2}=T_{1} / e^{4 \pi \nu}=11.3844 \mathrm{~N}$ is shown in Figure 9. It is almost stable which agrees with the analytical prediction greatly.

\section{Simulation of Arresting Gear System}

Making use of the modeling methodology developed in the Section 2, we build a full-scale MK7 type hydraulic arresting gear system with parameters shown in Table 1. To determine the mesh size of the cables in arresting gear system, we build a simple supported cable with same physical and geometrical parameters of the purchase cable as shown in Figure 10. It is subject to impact with initial velocity $20 \mathrm{~m} / \mathrm{s}$. The cable is uniformly meshed by 300 elements and 600 elements, respectively. The maximum displacement difference between these two meshes at center point within the first second is $1.3 \%$, and the maximum difference of stress is $4.1 \%$. Since 


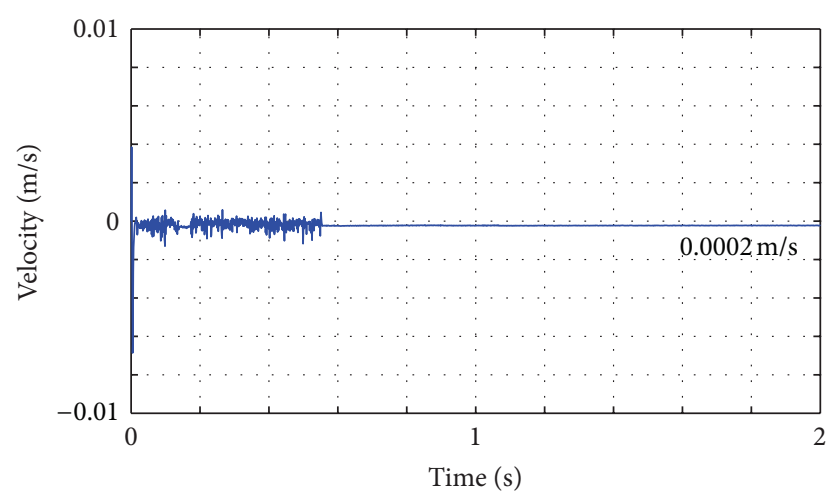

Figure 9: The velocity of the cable node.

TABLE 1: Parameters of the arresting gear system.

\begin{tabular}{lccc}
\hline Physical parameters & Symbol & Unit & Value \\
\hline Aircraft mass & $m_{a}$ & $\mathrm{~kg}$ & 25,000 \\
Landing speed & $V_{a}$ & $\mathrm{~m} / \mathrm{s}$ & 65 \\
Sheave mass & $m_{p}$ & $\mathrm{~kg}$ & 277.8 \\
Yong's modulus & $E_{c}$ & $\mathrm{~Pa}$ & $2 \times 10^{11}$ \\
Cable density & $\rho_{c}$ & $\mathrm{~kg} / \mathrm{m}^{3}$ & $7.8 \times 10^{3}$ \\
Damping ration & $\beta_{c}$ & - & $1 \times 10^{-6}$ \\
Stiffness coefficient & $K$ & $\mathrm{~N} / \mathrm{m}$ & $3 \times 10^{8}$ \\
Damping coefficient & $C$ & $\mathrm{~N} \cdot \mathrm{s} / \mathrm{m}$ & $1 \times 10^{4}$ \\
Collision index & $e$ & - & 1 \\
Friction coefficient & $v$ & - & 0.02 \\
\hline
\end{tabular}

both the displacement and stress converged to acceptable accuracies, we decided to use 300 elements every 30 meters to mesh the cables in arresting gear system.

In the following context, the aircraft landing along the runway centerline and offset the centerline will be simulated and analyzed, respectively. The acceleration, velocity, and position of aircraft and the stress within rope are presented. And the functionalities of cable anchor damper and damper sheave installation on the rope stress are analyzed in detail.

4.1. Landing along Centerline of Runway. At the most ideal situation, the landing aircraft velocity is parallel to the runway and the anchor hooks the cable right at the middle point. In this case, the response of aircraft from the initial arrestment to stop and the snapshots of the whole system are shown in Figure 11. It takes about $105 \mathrm{~m}$ and 3.52 seconds to decelerate the velocity from full speed to zero. And then the aircraft is pulled back a little because of the retraction force generated from the elastic energy stored in deck pendant.

The acceleration vibrates severely initially then stays around $2 \mathrm{~g}$ for a while and reduces to zero finally. Based on this, we divide the arresting process into three stages: (1) capture shock stage, (2) effective arresting stage, and (3) backward stage. The first stage is caused by the impact between tailhook and deck pendent which happened at $0.016 \mathrm{~s}$ because of the initial gap between them. It contains three acceleration peaks, and the maximum one is $3.98 \mathrm{~g}$ which is also the maximum acceleration of the whole arresting process. The character of entering the second stage is that the vibration of impact diminished, and acceleration gots stable. It ends when the velocity reduces to zero, and the elastic force generated by deck pendent leads to the third backward stage.

It is interesting to note that the stress at the middle of deck pendent, shown in Figure 12, performs similar to the acceleration of the aircraft. It also contains three peaks at the initial stage. It runs to slowly varying stage. This similarity implies that the acceleration of aircraft comes from the stress of cable at contact point. The stress gets its first peak point A once the impact between tailhook and cable happens. Then the stress wave generated by this shock propagates toward and reaches the deck sheave at $0.128 \mathrm{~s}$; it is reflected backward and results in the second stress peak B immediately after superposing with the original propagating one. When the backward wave reaches tailhook again at $0.197 \mathrm{~s}$, its reflection and superposition result in the third stress peak $C$ with value of 609.3 MPa which is also the maximum stress during the whole arresting process. Later, the stress wave decays, and the hydraulic force mainly generated by constant runout valve leads to a smooth variable period of stress. We will see later that the decay of the stress wave is caused by the absorbing effect of damper sheave installation and cable anchor damper.

4.2. Stress in Cable at Different Locations. Five points are chosen as the detection points on the deck pendant and purchase cable of the arresting gear system, as is shown in Figure 13. A is the middle point of the deck pendant; $\mathrm{B}$ is an arbitrary point in the purchase cable; $C$ and $D$ are the arbitrary two points in the purchase cable between crosshead and fixed sheaves where $\mathrm{C}$ is close to point $\mathrm{B}$ and $\mathrm{D}$ is close to point $\mathrm{E}$; $\mathrm{E}$ is an arbitrary point in the cable anchor installation. The results show that the maximum along the whole cable happens at the middle point of deck pendent. Each stress at the second stage from point $\mathrm{A}$ to $\mathrm{E}$ varies a little due to the friction between cable and sheaves but shares the same smooth varying process. At the first stage, the point $\mathrm{E}$ which is far away from contact point is not notable, influenced by the impact between deck pendent and cable.

4.3. Functionality of Damper Sheave Installation and Cable Anchor Damper. To understand and evaluate the functionality of damper sheave installations (DSI) and cable anchor damper (CAD), we build four models with parameters listed in Table 1, and the only difference is: (1) remove DSI and CAD, (2) remove CAD only, (3) remove DSI only, or (4) keep both DSI and CAD. The stresses at the middle of deck pendent with these four cases are shown in Figure 14.

Figure 14 shows the following cases.

(1) Without any damper, the stress of cable vibrates violently not only at the first stage but also at the second stage as shown by the red dashed line, and the maximum stress reaches $808 \mathrm{MPa}$.

(2) After installing DSI only, the maximum stress at the first stage is reduced by 24.6 percent to $609 \mathrm{MPa}$, and 

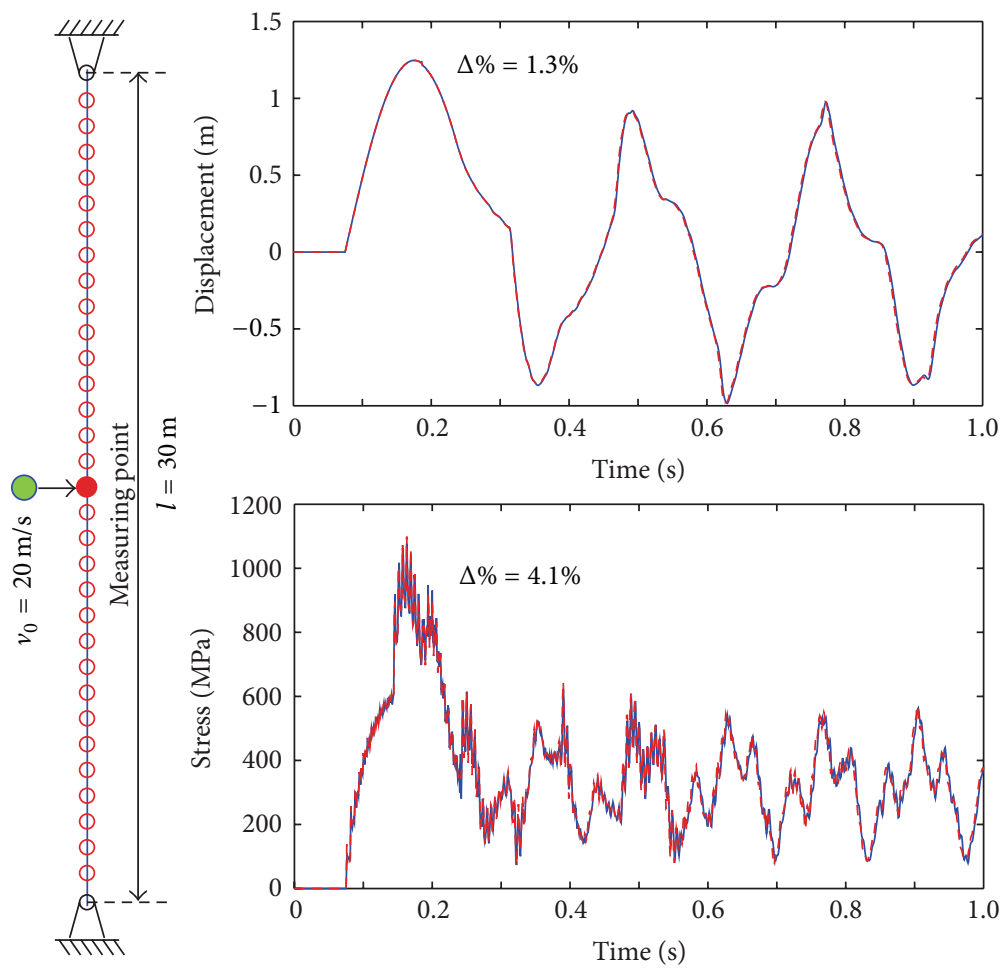

○ Node

- 300 nodes

-- - 600 nodes

Figure 10: The mesh convergence test.
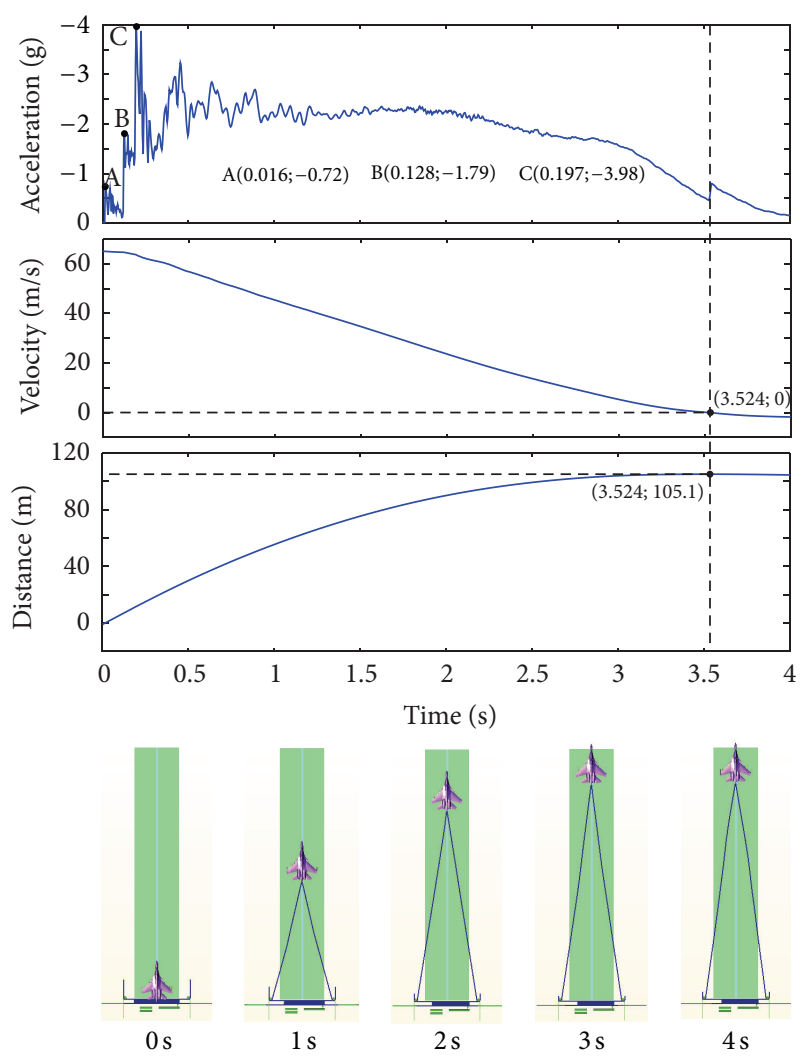

Figure 11: Movement of aircraft.

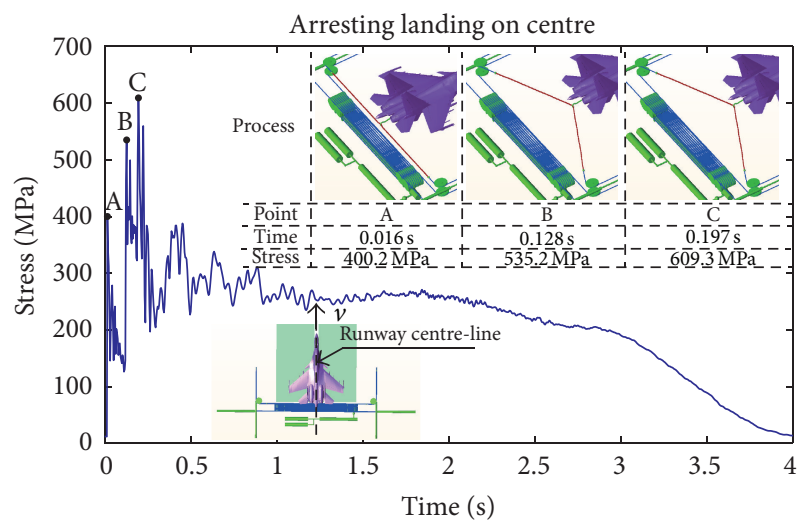

FIGURE 12: Stress of cable in the process of landing along center.

the stress vibration at the second stage is reduced greatly although it still exists.

(3) After installing CAD only, the maximum stress at the first stage is the same as without any damper, but the stress vibration at the second stage almost vanished as shown by the black dashed line.

(4) After installing both DSI and CAD, the first stage maximum stress is reduced to $609 \mathrm{MPa}$, and the second stage vibration is also vanished as shown by the pink solid line. 


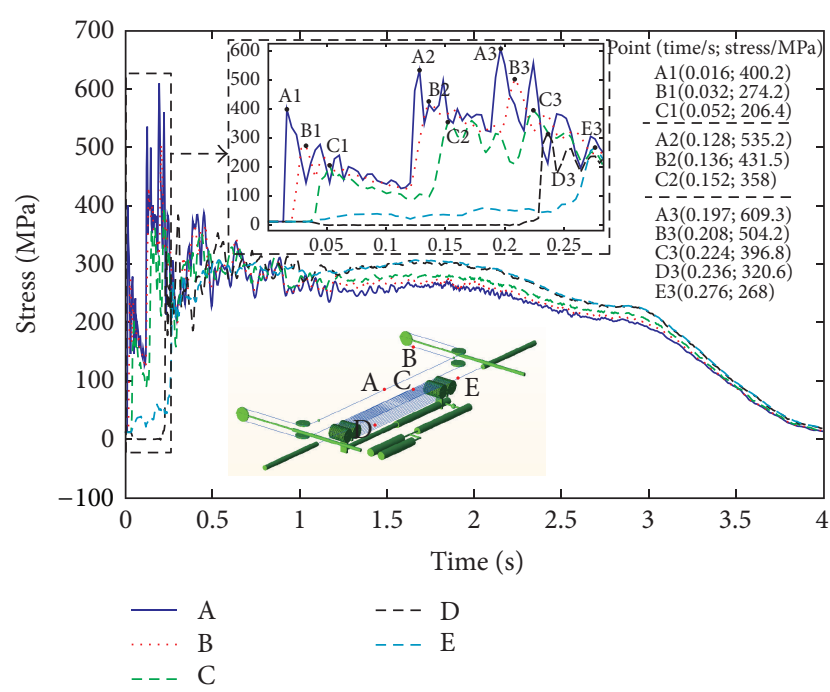

Figure 13: Stress of cable at different locations.

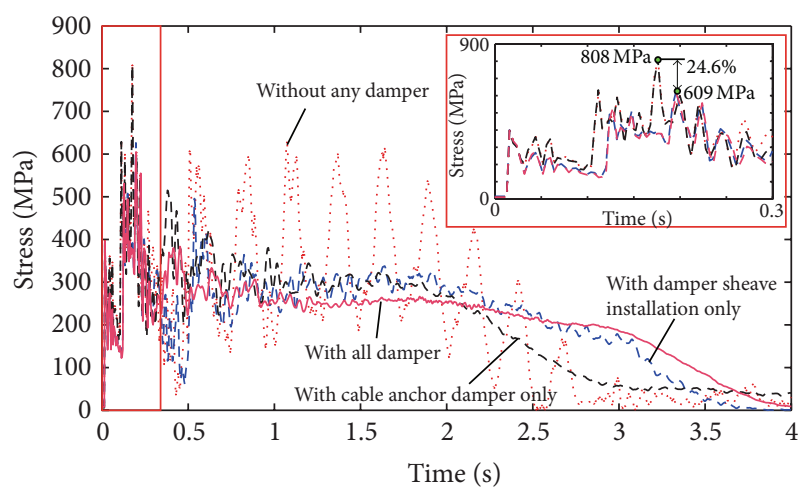

Figure 14: Cable stress of four cases at time domain.

Through the above analysis, we conclude that the CAD is far away from the tailhook and it can not generate any effect on the first shock stage, while the DSI is effective at both the first and the second stages. Besides, it is also inspirational to check the cable stress at frequency domain despite that the dynamic behavior of the arresting system keeps changing during arresting process. The stress at time range $[0.5,2.5]$ is analyzed via fast Fourier transformation (FFT); then the frequency resolution is $0.5 \mathrm{~Hz}$ since the sampling time lasts two seconds. As shown in Figure 15, there are three sharp sparks at $3.75 \mathrm{~Hz}, 7.5 \mathrm{~Hz}$, and $10.75 \mathrm{~Hz}$ when no damper is installed at all. This corresponds to the significant stress vibration in time domain. Further, it is interesting to note that the frequencies of second and third sparks are roughly two and three times that of the first spark. This behavior is very similar with the eigenfrequency character of a tensioned string. In fact, the cable length from deck sheave to fixed shave is always $25 \mathrm{~m}$ during the whole arresting process, and the stable stress within cable is around $300 \mathrm{Mpa}$ from Figure 13. Therefore, the fundamental frequency of this tensioned string is

$$
f_{0}=\frac{\sqrt{\sigma / \rho}}{2 l}=\frac{\sqrt{300 \times 10^{6} / 7860}}{2 \times 25}=3.9 \mathrm{~Hz}
$$

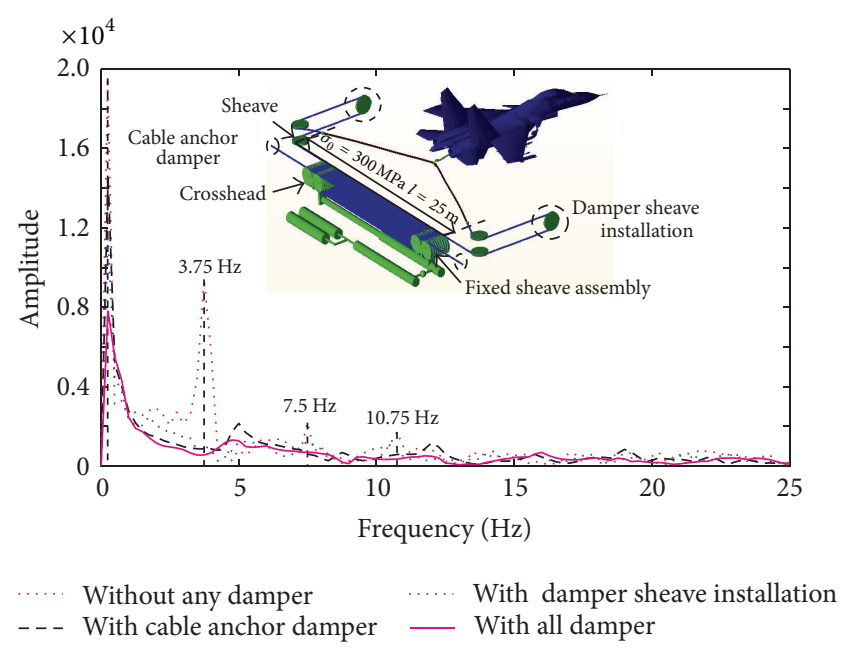

FIgURE 15: Cable stress of four cases at frequency domain.

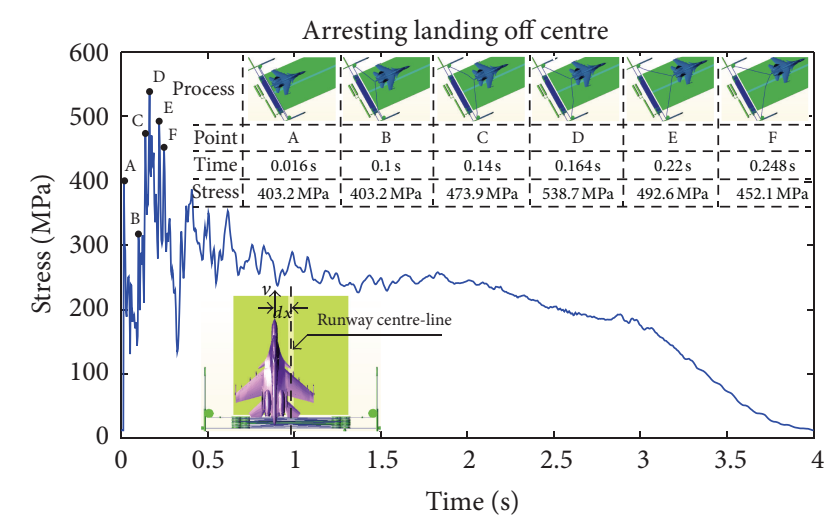

FIGURE 16: Stress of cable in the process of landing off center.

which agrees with the frequency of the first spark $3.75 \mathrm{~Hz}$ considering the frequency resolution of FFT. In addition, these sparks vanished after installing CAD or DSI since the damping force absorbed the vibration in cables severely.

4.4. Landing off Centerline of Runway. Now we discuss the situation of that the aircraft velocity is parallel with the runway centerline, while the landing point is $0.5 \mathrm{~m}$ left off the centerline. In this case, the stress at the middle point of the deck pendant during the whole process is shown in Figure 16.

It is shown from the simulation result that the deck pendant has 5 stress peaks at the first stage. The first is caused by the instant collision between the arresting hook and the deck pendant at $0.016 \mathrm{~s}$, and the second peak is caused by the reflection and superposition from the left deck sheave of the stress wave after it reached there. However, the third peak is caused by the reflection and superposition from the right deck sheave of the stress wave after it reached there. The fourth one is caused by the superposition of the transverse wave at the position of the arresting hook of the aircraft after it is reflected from the left deck sheaves. The fifth one is caused by the superposition of the transverse wave at the position of 
the arresting hook of the aircraft after it is reflected from the right deck sheaves.

Compared with along centerline landing case, we got five peak values here instead of three. And the maximum stress here is $539 \mathrm{MPa}$ which is smaller than $609 \mathrm{MPa}$ in case of along centerline. This means that the offline landing case could a little bit reduce the stress peak.

\section{Conclusion}

The arresting process is a complicated coupling dynamics between rigid bodies, flex bodies, and hydraulic units. It is strong nonlinear and involves both a transient wave propagation process in rope and a smooth decelerating of aircraft. This fact discounts the calculate efficiency of explicit method since the former process needs small time-step to capture the propagation events, while the later one needs large time-step to speed up. To solve this problem, in this paper, we proposed to build a multibody dynamic model of a full-scale MK7 type arresting gear system. And the resulting governing algebraic and differentiation equations are solved through a timevariable implicit backward differentiation formula. In the model, a kind of new cable element that is capable of describing the arbitrary large displacement and rotation in threedimensional space is developed to model the wire cables and damping force is used to simulate the effect of hydraulic system.

Dynamic simulation shows that the cable stress is dominated by the propagation, refection, and superposition of stress waves during the early stage of the arresting process, and later the shock is quickly dissipated by the damper sheave installations and cable anchor dampers. Simulation results repeat that maximum stress value happens when the stress wave is reflected and superposed between the deck sheaves. And the maximum stress in the off centerline landing case is a little bit smaller than the along centerline landing case. In addition, the multibody approach and arresting gear system model proposed here also provide an efficient way to design and optimize the whole mechanism.

\section{Nomenclature}

$\mathbf{q}_{\text {rigid }}$ : The generalized coordinate of rigid body

$\mathbf{r}_{\text {rigid }}$ : Cartesian coordinate of rigid body's position

$\lambda_{\text {rigid }}$ : Euler parameter of rigid body's attitude

$\mu_{k}: \quad$ Lagrange multiplier

$\Phi_{k}: \quad$ Constraint equations

$m_{i}$ : $\quad$ Mass tensor of the $i$ th rigid body

$\mathbf{J}_{i}$ : Inertia tensor of the $i$ th rigid body

$\mathbf{F}_{i}$ : The generalized force

$\mathbf{q}_{e}$ : The generalized elemental coordinates of cable

$\mathbf{f}(s, t)$ : Concentrated external load on cable element

$\varepsilon: \quad$ The normal strain of the cable element

E: The Yong's modulus of material

$\beta$ : $\quad$ The damping ratio of material $\sigma: \quad$ The stress of cable

$\mathbf{N}$ : The shape function of the cable element

r: The position vector of cable node

$\mathbf{M}_{e}$ : The elemental mass matrix of cable element

$\mathbf{K}_{e}$ : The elemental stiffness matrix of cable element

$\mathbf{F}_{e}: \quad$ The generalized force of cable element

$\mathbf{f}_{c}$ : The contact force

$\mathbf{Q}_{p}$ : The generalized collision force

$s_{n}$ : The element parameter of the detecting point

$\mathbf{f}_{n}$ : The force exerted on the detecting point

$F_{s}$ : The oil damping force in damper sheave installation

$k_{s}$ : The equivalent damping coefficient of the oil in damper sheave installation

$v_{s}: \quad$ The velocity of the piston in damper sheave installation

$u_{s}$ : The displacement of the piston in the sheave damper installation

$A_{s}: \quad$ The cross section area of the piston in damper sheave installation

$P_{s}: \quad$ The pressure of the gas in damper sheave installation

$\gamma: \quad$ The adiabatic coefficient of the gas

$T_{c}$ : The force of the cable anchor damper

$A_{c}$ : The cross section area of piston of the cable anchor damper

$m_{c}$ : The mass of the piston of the cable anchor damper

$P_{c}: \quad$ The pressure of the oil in the cylinder of the cable anchor damper

$P_{1}$ : The hydraulic pressure of the oil in the main engine cylinder

$A_{1}: \quad$ The effective cross section area of the ram

$m_{1}$ : The mass of the ram and crosshead

$v_{1}$ : The sliding velocity of the ram

$F_{1}$ : The force applied on the ram by the arresting rope

$\mu$ : The damping ratio of the hydraulic oil

$c_{d}: \quad$ The coefficient of the hydraulic oil

$A_{2}$ : The cross section area of the hole of the throttle

$P_{2}$ : The pressure of the energy accumulator

$x: \quad$ The position of the valve stem

$\phi: \quad$ Half of the top angle of valve stem

$d: \quad$ The diameter of the valve

$\alpha: \quad$ The rotation angle of the cam

$k_{1}, k_{2}$ : The functions describing the shape of the surface of the cam

$x_{0}$ : Initial area of the throttle

$k_{m}$ : The weight adjusting coefficient

$u: \quad$ The position of the crosshead

$k_{u}$ : The transfer coefficient between crosshead position and the rotation angle of cam

q: Generalized coordinates of the whole arresting system. 


\section{Acknowledgments}

This work is partially supported by a China Postdoctoral Science Foundation (2012M510417). And the authors would also like to thank Dr. Jiawei He for his kind help on visualizing simulation results. The anonymous reviewers also provided some very useful suggestions on improving this paper; it is highly appreciated.

\section{References}

[1] Z. Chengbang, "Aircraft arrester on carrier and its key technique," Weapons Spectacle, pp. 75-79, 2010.

[2] W. Haizhen, "Aircraft carrier," Ordnance Knowledge, no. 8A, pp. 45-47, 2011.

[3] http://www.militaryy.cn/html/73/n-40773.html.

[4] W. Billec, The Effect of Deck Span Upon Arresting Gear Performance, DTIC Document AD-813761, 1967.

[5] Z. Zhuokun, Dynamics analysis for carrier-based aircraft yawing and off-center arresting [M.S. thesis], Graduate school College of Aerospace Engineering; Nanjing University of Astronautics, Nanjing, China, 2010.

[6] Z. Zhuokun, N. Hong, Y. Hao, and W. Junlin, "Dynamics analysis for aircraft arresting yawing and off-center," Advance in Aeronautical Science and Engineering, vol. 1, no. 4, pp. 327-332, 2010.

[7] D. Mikhaluk, L. Voinov, and A. Borovkov, "Finite element modeling of the arresting gear and simulation of the aircraft deck landing dynamics," in Proceedings of the 7th European LS-DYNA Conference, Copyright by DYNAmore GmbH; Comp; MechLab of St. Petersburg State Polytechnical University, St. Petersburg, Russia, 2009.

[8] Z. Xinyu, Dynamics analysis and simulation of arresting cable [M.S. thesis], Control Theory and Control Engineering; Harbin Engineering University, Harbin, China, 2011.

[9] L. Lihua, W. Chen, and X. Panpan, "Dynamic analysis of aircraft arresting gear based on finite element method," in Proceedings of the International Conference on System Science, Engineering Design and Manufacturing Informatization (ICSEM '11), pp. 118121, Guiyang, China, October 2011.

[10] L. Lihua, W. Chen, and X. Panpan, "Study on the Dynamics Performance of Aircraft Arresting CableActa Aeronautical et Astronautical Sinica," http://www.cnki.net/kcms/detail/11.1929.V, 20120913.1126.007.html.

[11] J. Argyris, "An excursion into large rotations," Computer Methods in Applied Mechanics and Engineering, vol. 32, no. 1-3, pp. 85-155, 1982.

[12] H. Cheng and K. C. Gupta, "An historical note on finite rotations," American Society of Mechanical Engineers, vol. 56, no. 1, pp. 139-145, 1989.

[13] A. A. Shabana, "Definition of the slopes and the finite element absolute nodal coordinate formulation," Multibody System Dynamics, vol. 1, no. 3, pp. 339-348, 1997.

[14] A. A. Shabana and R. Y. Yakoub, "Three dimensional absolute nodal coordinate formulation for beam elements: theory," Journal of Mechanical Design, Transactions of the ASME, vol. 123, no. 4, pp. 606-613, 2001.

[15] L. Mingwan and L. Xuefu, Foundations of Elasticity, Chapter 3, Tsinghua University Press and Springer, Beijing, China, 2nd edition, 2001.
[16] L. Yongtang, L. Bufang, and G. Yuzhuo, Modeling and Simulation of the Hydraulic System, Chapter 2, Metallurgical Industry Press, Beijing, China, 2003.

[17] L. F. Shampine and M. W. Reichelt, “The MATLAB ODE suite," SIAM Journal on Scientific Computing, vol. 18, no. 1, pp. 1-22, 1997.

[18] E. Hairer and G. Wanner, Solving Ordinary Differential Equations. II: Stiff and Differential-Algebraic Problems, vol. 14 of Springer Series in Computational Mathematics, Chapter 5, Springer, Berlin, Germany, 2nd edition, 1996. 


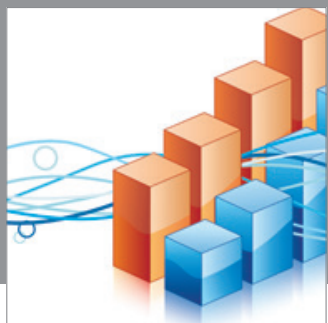

Advances in

Operations Research

mansans

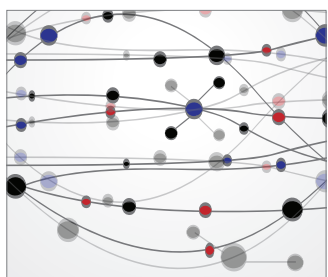

The Scientific World Journal
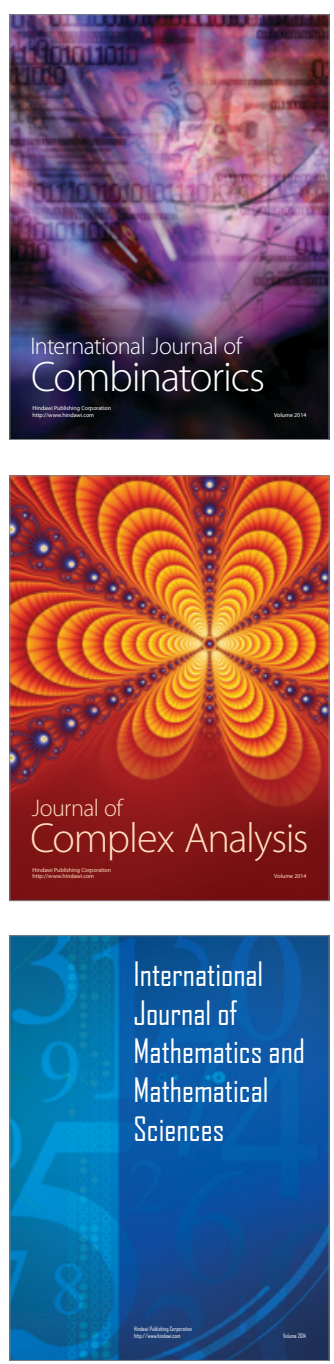
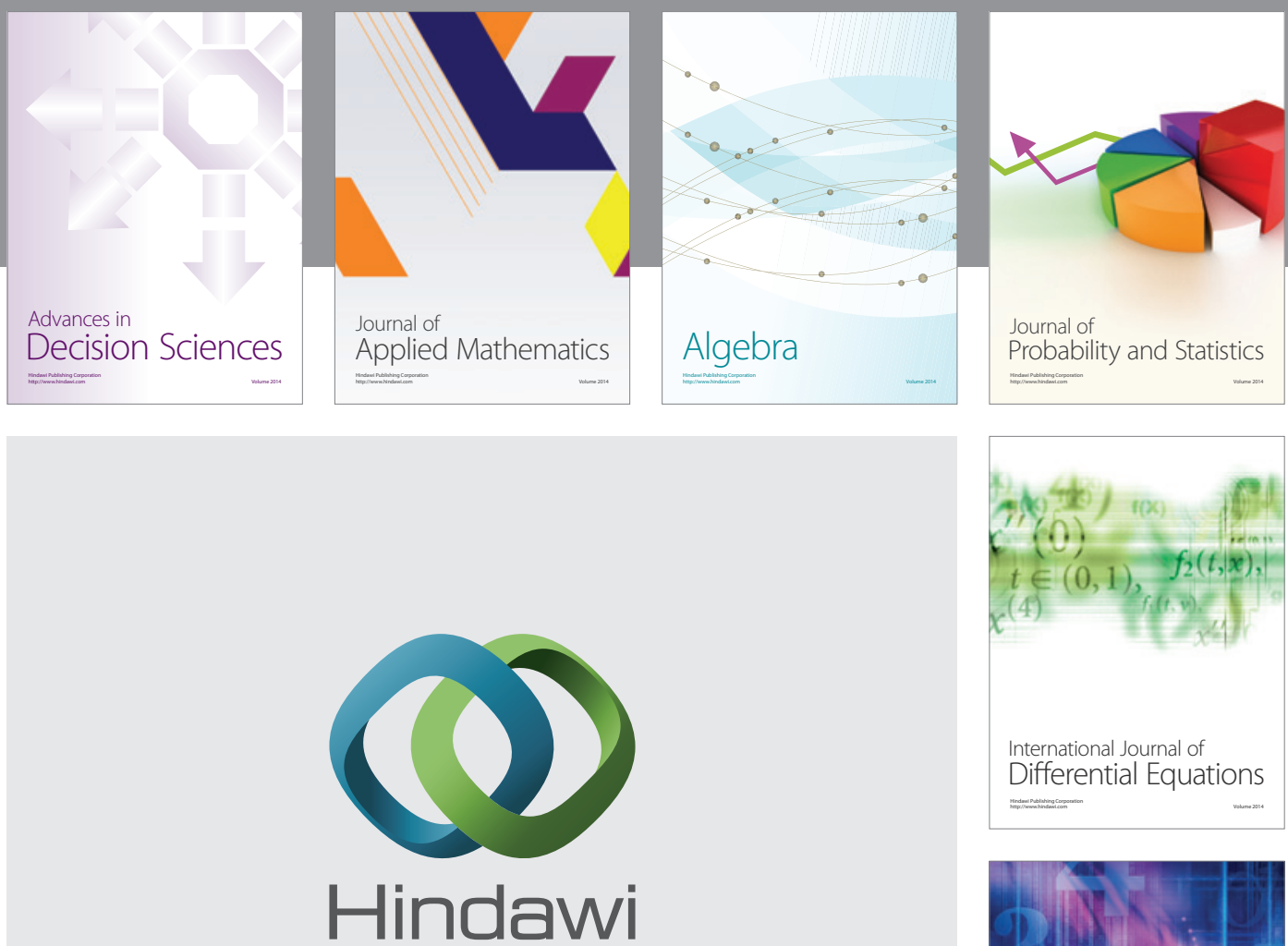

Submit your manuscripts at http://www.hindawi.com
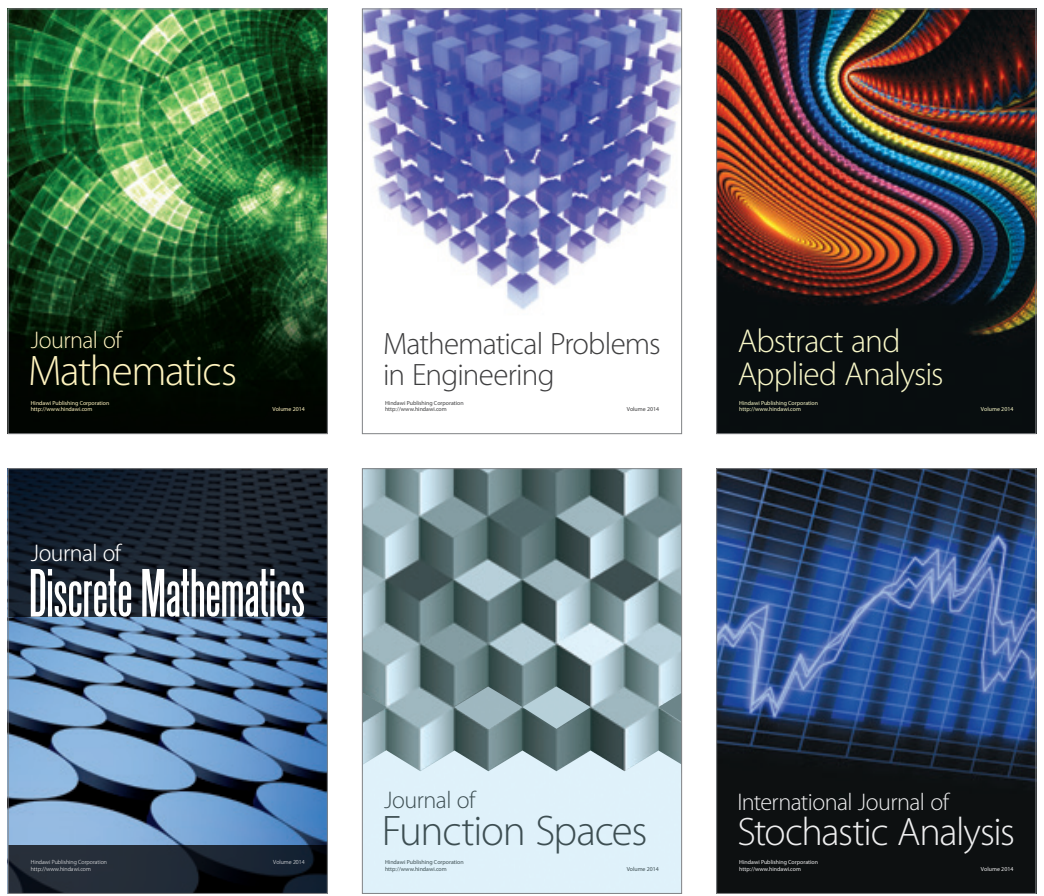

Journal of

Function Spaces

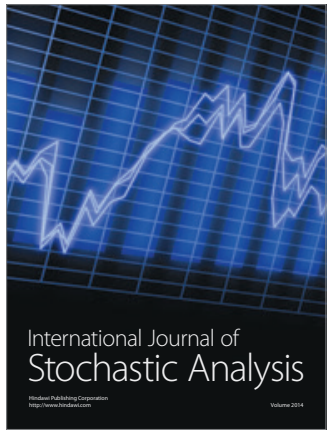

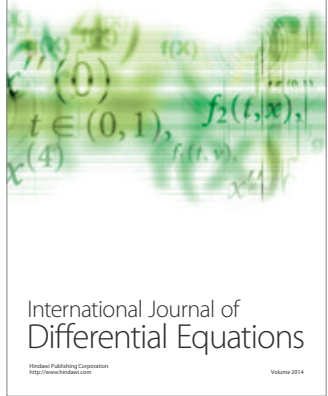
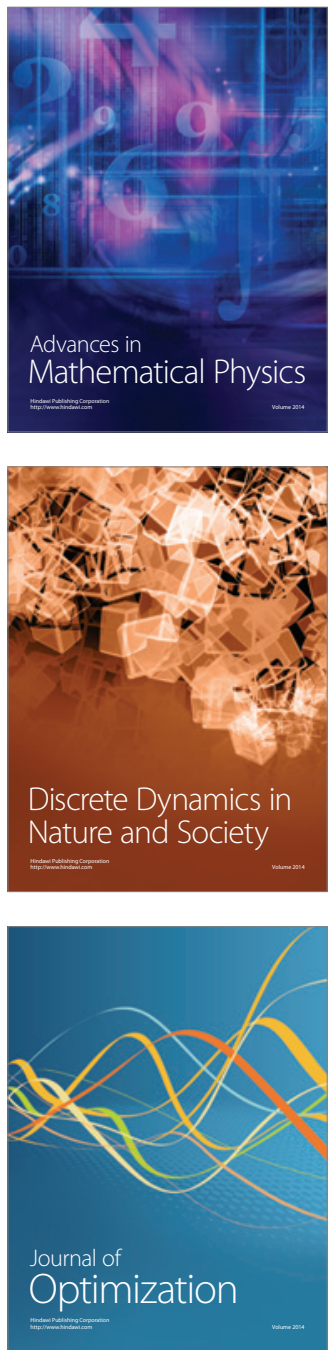